Pacific

Journal of

Mathematics

DIVERGENT SOLUTIONS OF THE HEAT EQUATION: ON AN ARTICLE OF LUTZ, MIYAKE AND SCHÄFKE

WERNER BALSER 


\title{
DIVERGENT SOLUTIONS OF THE HEAT EQUATION: ON AN ARTICLE OF LUTZ, MIYAKE AND SCHÄFKE
}

\author{
WERNER BALSER
}

In this article, we generalize results of Lutz, Miyake and Schäfke concerning summability of formal solutions of the Cauchy problem for the complex heat equation. In particular, we show that the type of summability depends on the given initial condition.

\section{Introduction.}

In detail, we will be concerned with the following problem for the complex heat equation (in two complex variables $\tau$ and $z$ ):

$$
u_{\tau}(\tau, z)=u_{z z}(\tau, z), \quad u(0, z)=\hat{\varphi}(z),
$$

where for the time being $\hat{\varphi}(z)=\sum \varphi_{n} z^{n}$ may denote a power series which may or may not have positive radius of convergence. A straightforward (formal) computation shows that (0.1) has a unique formal power series solution (in two variables) which may be written as

$$
\hat{u}(\tau, z)=\sum_{0}^{\infty} \frac{\hat{\varphi}^{(2 n)}(z)}{n !} \tau^{n} .
$$

D.A. Lutz, M. Miyake and R. Schäfke [5] have studied the above problem, assuming $\hat{\varphi}(z)$ convergent (for $|z|<r$, say, with $r>0$ ) to the analytic function $\varphi(z)$ (a copy of this article may be obtained, e.g., from the author of the present one). They showed that $(0.2)$ (with $\varphi(z)$ instead of $\hat{\varphi}(z)$ ) is one-summable in a direction $d$ if and only if $\varphi(z)$ can be analytically continued to infinity in directions $d / 2$ and $\pi+d / 2$, and is of exponential size at most two when going to infinity in these directions; by this we mean [3] that for sufficiently large constants $c_{j}$ we have

$$
|\varphi(z)| \leq c_{1} \exp \left(c_{2}|z|^{2}\right) .
$$

Here, we obtain analogous results for more general initial data. In particular, we shall show that the type of summability for the formal solution (0.2) varies with the given initial condition $\hat{\varphi}(z)$, which need not even be analytic at the origin, but may be a formal series with certain summability properties. The results for divergent initial data, however, are still not fully satisfactory, so Section 2 of this paper is slightly sketchy in nature. 
In the discussion to follow, we shall consider vectors $k=\left(k_{1}, \ldots, k_{\nu}\right)$ and $d=\left(d_{1}, \ldots, d_{\nu}\right)$ characterizing the type of (multi-)summability. A reader who is not familiar with the theory of multisummability but still wants to get the flavour of this article, may restrict himself to the situation of $\nu=1$, hence $k=k_{1}, d=d_{1}$, which is still meaningful and non-trivial. For more ambitious readers, we refer to, e.g., [3] for a self-contained presentation of the theory of multisummability, and emphasize that whatever results and notation concerning multisummability for $\nu>1$ are used in this article can be found there.

Acknowledgement. I am grateful to D.A. Lutz and M. Miyake who, during my stay at SDSU, San Diego, introduced me to their common results in this area and inspired me to write this paper. I also wish to thank the referee for valuable hints improving readability of this article.

\section{Convergent initial conditions.}

Here we consider the special situation of (0.1) where the initial condition $\hat{\varphi}(z)$ is a power series having a positive radius of convergence, and we shall denote the function defined by this series as $\varphi(z)$. Instead of the formal solution (0.2) we then consider the semiformal solution

$$
\check{u}(\tau, z)=\sum_{0}^{\infty} \frac{\varphi^{(2 n)}(z)}{n !} \tau^{n}, \quad|z|<R .
$$

Note that this still is a formal series in the time-variable $\tau$, so the question of its summability properties (for a fixed value of the space-variable $z$ ) remains. However, observe that summability of (1.1) should better produce a function which is analytic in both variables, so we will always make the following requirements which guarantee locally uniform summability in the variable $z$ for $z$ close to the origin:

Definition. Given any $d$ and $k>0$, we say that (1.1) near the origin is locally $k$-summable in direction $d$, iff there exist positive constants $\rho, R, C, K, \alpha$ such that the following holds for all $z$ with $|z|<\rho$ :

The formal Borel transform

$$
v(s, z)=\sum_{0}^{\infty} \frac{\varphi^{(2 n)}(z)}{n !} \frac{s^{n}}{\Gamma(1+n / k)}
$$

converges for $|s|<R$, and moreover, $v(s, z)$ can be analytically continued with respect to $s$ into the sector $S(d, \alpha)$ of opening $\alpha$ and bisected by $\arg s=$ $d$ (and of infinite radius), and

$$
|v(s, z)| \leq C \exp \left(K|s|^{k}\right), \quad s \in S_{d} .
$$


Note that this definition assures uniform convergence with respect to $z$ of the Laplace integral in $\tau$ used to represent the sum of $\check{u}(\tau, z)$. Similarly, one can define local multisummability in some multi-direction: In this case, $k$ and $d$ will be vectors $\left(k_{1}, \ldots, k_{\nu}\right)$ resp. $\left(d_{1}, \ldots, d_{\nu}\right)$, with $k_{1}>\ldots>k_{\nu}>0$, and reals $d_{j}$ restricted by

$$
\left|d_{j}-d_{j-1}\right| \leq \pi\left(1 / k_{j}-1 / k_{j-1}\right), \quad 2 \leq j \leq \nu .
$$

For the complete definition of multisummability we refer to [3]; for this article it suffices to know that, according to [1], every multisummable formal power series can be split into a sum of series, each of which is $k_{j}$-summable in direction $d_{j}$, for some $j$.

For $z$ in some (small) open set, let positive constants $C, K, \tilde{k}$, independent of $z$, be given so that

$$
\left|\varphi^{(2 n)}(z)\right| \leq C K^{n} \Gamma(1+n / \tilde{k}), \quad n \geq 0 .
$$

Generally this holds for $\tilde{k}=1 / 2$, and for this case one-summability of (1.1) was investigated in detail in [5] (see the introduction for a description of their result). However, if $\varphi(z)$ is entire, then (1.4) may hold for larger values of $\tilde{k}$, and is equivalent to $\varphi(z)$ being of exponential size at most $\kappa=2 \tilde{k} /(2 \tilde{k}-1)$. In particular, the case of $\tilde{k}=1$ is equivalent to convergence of (1.1), so for what follows we may restrict to $1 / 2 \leq \tilde{k}<1$.

Theorem 1. Under the above assumptions on the initial data $\hat{\varphi}(z)=$ $\sum \varphi_{n} z^{n}$, let any multisummability-type $k=\left(k_{1}, \ldots, k_{\nu}\right)$ with $1 / k_{\nu}=1 / \tilde{k}-$ 1 , and a multi-direction $d=\left(d_{1}, \ldots, d_{\nu}\right)$, admissible with respect to $k$, be given. Then the following statements are equivalent:

a) Near the origin, the formal series (1.1) is locally $k$-summable in direction $d$.

b) For $j=0$ and $j=1$, the formal series

$$
\hat{\psi}_{j}(z)=\sum_{0}^{\infty} \varphi_{2 n+j} \frac{(2 n+j) !}{n !} z^{n}
$$

are $k$-summable in direction $d$.

c) The formal series

$$
\hat{\psi}^{ \pm}(z)=\sum_{0}^{\infty} \varphi_{n} \Gamma(1+n / 2)( \pm z)^{n}
$$

both are $2 k$-summable in direction $d / 2$, with $2 k=\left(2 k_{1}, \ldots, 2 k_{\nu}\right)$ and $d / 2=\left(d_{1} / 2, \ldots, d_{\nu} / 2\right)$.

Proof. In case of a), we conclude that both $\check{u}(\tau, z)$ and $\partial_{z} \check{u}(\tau, z)$ near $z_{0}=0$ are $k$-summable in direction $d$. Taking $z=0$ and replacing $\tau$ by $z$, this implies b). From the definition of multisummability it is easily seen that b) 
is equivalent to $\hat{\psi}_{j}\left(( \pm z)^{2}\right)$ being $2 k$-summable in direction $d / 2$, and applying [3], p. 31 Ex. 5, together with results from [3], p. 78, on summability factors one can then conclude that this in turn is equivalent to $\mathrm{c}$ ). This leaves to conclude a) from either b) or c):

To do so, first note that according to [1], power series which are $k^{-}$ summable in direction $d$ can be written as a sum of (finitely many) series which each are $k_{j}$-summable in direction $d_{j}$, for some $j, 1 \leq j \leq \nu$, and conversely, sums of such series are $k$-summable in direction $d$. Using this, it is easy to conclude that we may restrict to the case of $\nu=1$, hence $k=k_{1}$. Next, observe that (formally) one can show

$$
\hat{u}(\tau, z)=\sum_{m=0}^{\infty} z^{m} \hat{\psi}_{m}(\tau),
$$

with $\hat{\psi}_{0}(\tau), \hat{\psi}_{1}(\tau)$ as in b), and for $m=2 \mu+j, \quad 0 \leq j \leq 1$ :

$$
\hat{\psi}_{m}(\tau)=\frac{\hat{\psi}_{j}^{(\mu)}(\tau)}{m !} .
$$

So every $\hat{\psi}_{m}(\tau)$ is $k$-summable in direction $d$, and if we let $\psi_{m}(\tau), \tau \in S$ denote its sum (for a sector $S$ which may be taken independent of $m$ ), then for every closed subsector $\bar{S} \subset S$ there exist constants $C, K$ with

$$
\left|\psi_{m}(\tau)\right| \leq C K^{m} \mu ! \Gamma(1+\mu / k), \quad \tau \in \bar{S}, m \geq 0 .
$$

Hence the series

$$
u(\tau, z)=\sum_{m=0}^{\infty} z^{m} \psi_{m}(\tau)
$$

converges uniformly (in two variables) for $|z|$ sufficiently small and $\tau \in \bar{S}$ (in fact, if $k>1$, i.e. $\varphi(z)$ entire, convergence takes place for every $z$ ). From this observation we may conclude that the function $u(\tau, z)$ has $\breve{u}(\tau, z)$ as its Gevrey asymptotic (in the variable $\tau$ ) of order $k=k_{1}$ in a sector of opening more than $\pi / k$, and this asymptotic is uniform with respect to $z$. This, however, implies a), which completes the proof.

Remark 1. It is worthwile observing that for $\tau \neq 0$ the series (1.6) in fact converges for every $z$, hence represents an entire function in the space variable which may be seen to be of exponential size at most two. This coincides with the classical result on the convergence of (1.1); compare for this the last remark in this section.

Remark 2. The formal series $\hat{\psi}_{j}(z)$ resp. $\hat{\psi}^{ \pm}(z)$ are explicitly related to the initial condition $\hat{\varphi}(z)$. Using acceleration and Laplace operators, one can explicitly reformulate a), b) in terms of transforms of the function $\varphi(z)$, but it is generally difficult to check a) or b) directly through investigation of 
$\varphi(z)$. Compare, however, the next remark for the most interesting special case of $\nu=1, k=k_{1}=1$.

Remark 3. The above theorem, in case of $\nu=1$ and $k=1$, essentially coincides with the result obtained in [5], since in this case one can see that a) or b) are equivalent to $\varphi(z)$ admitting analytic continuation into (small) sectors bisected by rays $\arg z=d$ and $\arg z=d+\pi$, and being of exponential size not more than two there. The proof we gave here is essentially different from the one in [5], and we should also like to mention the following simpler representation for the solution which involves an acceleration operator instead of the Laplace operator used in [5]: One can show for the sum $u(\tau, z)$ of the semiformal solution the formula

$$
u(\tau, z)=\tau^{-1 / 2} \int_{0}^{\infty(d)} \frac{\varphi\left(z+s^{1 / 2}\right)+\varphi\left(z-s^{1 / 2}\right)}{2} C_{2}\left((s / \tau)^{1 / 2}\right) d s^{1 / 2}
$$

where $C_{\alpha}(u)$ is an entire function serving as kernel in Ecalle's acceleration operators $[\mathbf{3}]$.

Remark 4. As follows from the proof of the above theorem, in case of $k_{\nu}>1$ we have summability of (1.1) for every $z$. For $k_{\nu}=1$, however, summability takes place only in a disc whose radius generally is much smaller than the radius of convergence of $\hat{\varphi}(z)$. One may analytically continue the sum with respect to $z$ by allowing in the integral transformations used in the summation process that the paths of integration, in dependence on the value of $z$, are no longer straight lines but curves. We shall not go into details about this here.

Remark 5. Since $k$-summability in all directions is equivalent to convergence, we obtain as a corollary of the above theorem that convergence of (1.1) is equivalent to the initial condition being an entire function of exponential size at most two. This, however, is a well known classical result.

\section{Divergent initial conditions.}

Despite of the fact that it appears natural to require the initial condition $\hat{\varphi}(z)$ to converge, we see that the statement of the above theorem makes good sense even when this is not so, but instead $\hat{\varphi}(z)$ is assumed to be multisummable of some type, and $\varphi(z)$ then denotes its sum. The proof we gave above, however, fails under this (weaker) assumption. To see whether the result nonetheless carries over, we first show:

Lemma 1. For any summability type $k$ and any multidirection $d$, admissible with respect to $k$, the following two statements are equivalent:

a) Near the origin, the semiformal solution $\check{u}(\tau, z)$ is locally $k=\left(k_{1}, \ldots\right.$, $\left.k_{\nu}\right)$-summable in direction $d=\left(d_{1}, \ldots, d_{\nu}\right)$. 
b) Near the origin, the semiformal series

$$
\check{u}_{1}(\tau, z)=\sum_{0}^{\infty} \frac{\tau^{n}}{\Gamma(1+n / 2)} \varphi^{(n)}(z)
$$

is locally $2 k=\left(2 k_{1} \ldots, 2 k_{\nu}\right)$-summable in directions $d / 2=\left(d_{1} / 2, \ldots\right.$, $\left.d_{\nu} / 2\right)$ and $\pi+d / 2=\left(\pi+d_{1} / 2, \ldots, \pi+d_{\nu} / 2\right)$.

Proof. Assume a) and conclude that then termwise differentiation with respect to $z$, followed by a fractional termwise integration with respect to $\tau$ may be used to find that

$$
\sum_{0}^{\infty} \frac{\tau^{n+1 / 2}}{\Gamma(3 / 2+n)} \varphi^{(2 n+1)}(z)
$$

near the origin is locally $k=\left(k_{1}, \ldots, k_{\nu}\right)$-summable in direction $d=\left(d_{1}, \ldots\right.$, $\left.d_{\nu}\right)$. Adding this series to $\check{u}(\tau, z)$ and replacing $\tau$ by $\tau^{2}$ we then conclude b). Conversely, from b) we conclude that both the odd and even part of (2.1) are locally $2 k$-summable in direction $d / 2$, and this is easily seen to imply a).

We now investigate condition b) of the previous Lemma in the case of $k_{\nu}<1$ : Taking $z=0$ (and then replacing $\tau$ by $z$ ), we obtain as a necessary condition that

$$
\hat{\psi}(z)=\sum \varphi_{n} \frac{\Gamma(1+n)}{\Gamma(1+n / 2)} z^{n}
$$

is $2 k$-summable in directions $d / 2$ and $\pi+d / 2$. To investigate sufficiency, we restrict to the case of $\nu=1$ (note that this can be done without loss of generality: According to an observation of B. Faber [4], we may introduce "artificial levels" into the summability type $k$ so that eventually $1 / k_{j}-$ $1 / k_{j-1}<2, \quad 2 \leq j \leq \nu$, and then apply a theorem in [2] to decompose $\hat{\psi}(z)$ into a sum of formal series which are $k_{j}$-summable in direction $d_{j}$, for $1 \leq j \leq \nu$; the artificially introduced levels are recognizable later on since they belong to directions $d_{j}$ which can be chosen arbitrarily within a corresponding interval). If $k=k_{1} \geq 1$, the results obtained in the previous section apply, so we may assume the opposite. Then $\hat{\varphi}(z)$, for $1 / \tilde{k}=(1 / k-$ $1) / 2$, is $\tilde{k}$-summable in directions $d / 2$ and $\pi+d / 2$. Moreover, the $\operatorname{sum} \varphi(z)$ is analytic in two sectors $S_{1}, S_{2}$ bisected by $\arg z=d / 2$ resp. $\arg (-z)=d / 2$, and both of opening larger than $\pi / \tilde{k}_{1}$ (and of infinite radius), and is of exponential growth not more than two in both sectors. For any closed subsector $\bar{S}$ of either $S_{1}$ or $S_{2}$, we then can find constants $C, K$ such that

$$
\left|\varphi^{(n)}(z)\right| \leq C K^{n} n ! \Gamma(1+n / \tilde{k}), z \in \bar{S}, n \geq 0 .
$$

(Observe that as long as $z \neq 0$, we have the much better estimate

$$
\left|\varphi^{(n)}(z)\right| \leq C_{z} K_{z}^{n} n !, n \geq 0,
$$


but the constants generally explode as $z \rightarrow 0$.) So for $1 / \kappa=1+1 / \tilde{k}=$ $(1 / k+1) / 2$, the series

$$
v(s, z)=\sum_{0}^{\infty} \frac{s^{n}}{\Gamma(1+n / \kappa)} \varphi^{(n)}(z)
$$

converges for sufficiently small values (of both variables). For $z \neq 0$ we even have that $v(s, z)$ is entire in $s$ and of exponential size not more than $\tilde{k}$, while for $z=0$ (because of $v(s, 0)=\sum \varphi_{n} s^{n} n ! / \Gamma(1+n / \kappa)$, which can be related to $\sum \varphi_{n} s^{n} / \Gamma(1+n / \tilde{k})$ using the Beta Integral) we have that $v(s, z)$ can be analytically continued to infinity in both directions $d / 2$ and $\pi+d / 2$ and is of the same exponential size. So we may apply the acceleration operator $\mathcal{A}_{1, \hat{k}}$ (with respect to the variable $s$ ) to $v(s, z)$, and the resulting function $\tilde{v}(s, z)$, for fixed $z$, satisfies

$$
\tilde{v}(s, z) \cong_{\tilde{k}} \sum_{0}^{\infty} \frac{s^{n}}{\Gamma(1+n)} \varphi^{(n)}(z) .
$$

For $z \neq 0$ this asymptotic even converges, so we find

$$
\tilde{v}(s, z)=\varphi(s+z) \text {. }
$$

So for fixed $z \in S_{1}$ (resp. $z \in S_{2}$ ) we have that $\tilde{v}(s, z)=\varphi(s+z)$ can be analytically continued (in the variable $s$ ) into a sector of opening more than $\pi / \tilde{k}$ and bisected by $\arg s=d / 2$ (resp. by $\arg s=\pi+d / 2$ ) and is of exponential growth not more than two there; hence in other words, $\breve{u}_{1}(\tau, z)$ is $k$-summable in direction $d / 2$ (resp. $\pi+d / 2$ ). However, it is easily seen that generally summability in the opposite direction fails, because we cannot analytically continue $\tilde{v}(s, z)$ in direction $\arg s=\pi+d / 2(\operatorname{resp} . \arg s=d / 2)$. So one can conclude that the only cases where condition b) can hold are the trivial ones where the series is actually convergent (hence $\varphi(z)$ entire of exponential size at most two).

Nonetheless, there is a way out of this dilemma by relaxing the definition of $k$-summability in the following perfectly natural way: We use a generalized notion of analytic continuation and at the same time allow paths of integration in acceleration operators which are not necessarily straight lines. As for analytic continuation we say as follows:

Definition. Suppose that $f(z)$ is analytic along a path $\gamma$ from $z_{0}$ to $z_{1}$ (and without loss of generality let $\gamma$ have a tangent line $\arg \left(z-z_{1}\right)=d$ at the endpoint). Moreover, assume that $f(z)$ has a formal power series expansion $\hat{f}\left(z-z_{1}\right)$ when we approach $z_{1}$ along the path $\gamma$. Then, in case of $\hat{f}\left(z-z_{1}\right)$ being multisummable of some type $k=\left(k_{1}, \ldots, k_{\nu}\right)$ in the multidirection $(d+\pi, \ldots, d+\pi)(\nu$-times repeated), we say that $f(z)$ can be analytically continued through the singular point $z_{1}$, and we regard the multisum of $\hat{f}(z-$ 
$z_{1}$ ) in the said direction (being analytic in a sector "opposite" of the path $\gamma$ ) as its analytic continuation.

Using this terminology, we see that $\tilde{v}(s, z)=\varphi(s+z)$ can now indeed be analytically continued with respect to $s$, for $z \in S_{1}$, through the singular point at $s=-z$ and then all the way to infinity in a sector bisected by $\arg s=\pi+d / 2$ and of opening more than $\pi / \tilde{k}$, satisfying the correct growth estimate to apply the acceleration operator $\mathcal{A}_{2,1}$ with path of integration being the straight line segment from $s=0$ to $s=-z$ followed by a ray $\arg (s+z)=\alpha$ within the above-mentioned sector. Altogether we may say that this means that for $\nu=1, k=k_{1}<1$ and $\hat{\psi}(z) 2 k$-summable in directions $d / 2$ and $\pi+d / 2$, the series $\check{u}_{1}(\tau, z)$ will not have the same type of (local) summability with respect to $\tau$, but instead may be said to be weakly $(2,2 k)$-summable in direction $(d / 2, d / 2)$ and $(\pi+d / 2, \pi+d / 2)$. Consequently, the semiformal solution $\check{u}(\tau, z)$ will be weakly $(1, k)$-summable in direction $(d, d)$.

The above rough argument shows that Theorem 1 does not directly generalize to divergent initial conditions. On the other hand, there is a way to cover such cases which involves relaxing the definition of summability in a suitable fashion - but it becomes clear from the above discussion that level one will always be singular whenever $z \neq 0$. We will not formally state this result as a theorem, since it is not entirely clear whether this situation of divergent initial conditions occurs "naturally" in applications. Compare, however, the end of the next section, where we will recover the same result using a different approach.

\section{Convergent initial conditions revisited.}

We now return to the situation of the initial condition being analytic in a (small) circle about the origin: While Theorem 1 characterizes the cases of the semiformal solution being multisummable of some type or other, there are definitely situations where this will not occur. For example, if $\varphi(z)$ cannot be analytically continued beyond the circle of convergence (assuming its radius being finite), we never have multisummability in any direction. So this leaves the question of interpreting the semiformal solution in such cases, e.g. by identifying a solution of the heat equation which naturally corresponds to $\check{u}(\tau, z)$ - whatever natural may mean here.

For this purpose, let $w$ be a fixed non-zero complex number and take $\varphi(z)=(w-z)^{-1}$. Then Theorem 1 implies that the corresponding semiformal solution is 1-summable in all directions except the one through the point $w$, and using the representation formula (1.7), one can show for the $\operatorname{sum} u(\tau, z)$ :

$$
u(\tau, z)=(w-z)^{-1} k\left(\tau(w-z)^{-2}\right)
$$


with

$$
k(z)=z^{-1 / 2} \int_{0}^{\infty(\alpha)} C_{2}\left((u / z)^{1 / 2}\right) \frac{d u^{1 / 2}}{1-u},
$$

where integration is along any ray other than the positive real axis. So $k(z)$ is a multi-valued analytic function with a single branchpoint at the origin and satisfies

$$
\begin{gathered}
k(z) \cong_{1} \sum_{0}^{\infty} \frac{(2 n) !}{n !} z^{n} \quad \text { in }-\pi / 2<\arg z<5 \pi / 2, \\
k\left(z \mathrm{e}^{2 \pi i}\right)-k(z)=-\pi i z^{-1 / 2} C_{2}\left(z^{-1 / 2}\right), \quad-\pi / 2<\arg z<\pi / 2 .
\end{gathered}
$$

Let now $\varphi(z)$ be any function analytic in $|z|<R$, for some $R>0$. Choose a number $a$ inside the disc $|z|<R$; for simplicity we restrict to positive real $a$, but the arguments below will generalize. For $z$ in the upper half plane and $\tau$ on the positive real axis, we define

$$
u_{+}(\tau, z)=\frac{1}{2 \pi i} \int_{-a}^{a} \frac{\varphi(w)}{w-z} k\left(\tau(w-z)^{-2}\right) d w
$$

(integrating along the real axis). The function then is a solution of the heat equation and can be analytically continued across the line segment $(-a, a)$ by deforming the path of integration to become a semicircle in the lower half plane. Restricting $z$ to the square with vertices $\pm a, \pm i a$, and letting $w$ vary along the lower semicircle, one may check that the set of $\tau$ with $-\pi / 2<\arg \left(\tau(w-z)^{-2}\right)<5 \pi / 2$ contains a sector $S_{z}$ bisected by the positive real axis and of an opening depending on $z$. If we choose the branch of $k\left(\tau(w-z)^{-2}\right)$ according to this specification of the argument, then interchanging integration and limit implies

$$
u_{+}(\tau, z) \rightarrow \frac{1}{2 \pi i} \int_{-a}^{a} \frac{\varphi(w)}{w-z} d w \quad \text { as } \tau \rightarrow 0 \text { in } S_{z}
$$

(integrating along the lower semicircle). Corrrespondingly, for $w$ on the upper semicircle, the set of $\tau$ with $-5 \pi / 2<\arg \left(\tau(w-z)^{-2}\right)<\pi / 2$ also contains the sector $S_{z}$, and if we define

$$
u_{-}(\tau, z)=\frac{1}{2 \pi i} \int_{-a}^{a} \frac{\varphi(w)}{w-z} k\left(\mathrm{e}^{2 \pi i} \tau(w-z)^{-2}\right) d w,
$$

then

$$
u_{-}(\tau, z) \rightarrow \frac{1}{2 \pi i} \int_{-a}^{a} \frac{\varphi(w)}{w-z} d w \quad \text { as } \tau \rightarrow 0 \text { in } S_{z}
$$

(integrating along the upper semicircle). Therefore,

$$
u(\tau, z)=u_{+}(\tau, z)-u_{-}(\tau, z)
$$

is a solution of the heat equation satisfying

$$
u(\tau, z) \rightarrow \varphi(z) \text { as } \tau \rightarrow 0 \text { in } S_{z} .
$$


Instead of a limit, it is easy to see that we have a full Gevrey-one asymptotic to the semiformal solution $\breve{u}(\tau, z)$; however, the opening of the sector $S_{z}$ is too small (less than or equal to $\pi$ ) to characterize the solution uniquely on the contrary, the solution so obtained clearly depends upon the (real) parameter $a$.

To see how this solution relates to the results of the earlier sections, observe

$$
k(z)=\int_{0}^{\infty(\beta)} C_{2}(u) \frac{d u}{1-z u^{2}}, \quad|\tau|<\pi / 4,
$$

showing that $k(z)$ remains bounded as $z \rightarrow \infty$ in arbitrary sectors. Hence instead of half circles, we can also integrate from $-a$ to $z$ and then to $a$, both times along straight line segments. Making the correct choices for $\arg (w-z)$, one then finds

$$
\begin{aligned}
u(\tau, z)= & \frac{1}{2 t^{1 / 2}}\left(\int_{z}^{a}-\int_{-a}^{z}\right) \varphi(w) C_{2}\left((w-z) t^{-1 / 2}\right) d w \\
= & \frac{1}{2 t^{1 / 2}}\left(\int_{0}^{(a-z)^{2}} \varphi\left(z+s^{1 / 2}\right) C_{2}\left((s / t)^{1 / 2}\right) d s^{1 / 2}\right. \\
& \left.+\int_{0}^{(a+z)^{2}} \varphi\left(z-s^{1 / 2}\right) C_{2}\left((s / t)^{1 / 2}\right) d s^{1 / 2}\right) .
\end{aligned}
$$

In cases where $\varphi(z)$ can be analytically continued to infinity along the real axis and behaves so that we may take the limit $a \rightarrow \infty$, we get a unique solution, and in this fashion one can recover the representation (1.7). Even if $\varphi(z)$ is not analytic near the origin, but in two sectors $S_{1}, S_{2}$ as in the previous section, we still can use (3.2) to define a solution (depending on $a$ ), and then take a limit as $a \rightarrow \infty$. Doing so, one then recovers the same results as in Section 2.

\section{References}

[1] W. Balser, A different characterization of multisummable power series, Analysis, 12 (1992), 57-65.

[2] - Addendum to my paper: A different characterization of multi-summable power series, Analysis, 13 (1993), 317-319.

[3] , From Divergent Power Series to Analytic Functions, Lecture Notes in Math., Vol. 1582, Springer-Verlag, 1994.

[4] B. Faber, Oral communication, May 1994, Conference in Lille. 
[5] D.A. Lutz, M. Miyake and R. Schäfke, On the Borel summability of divergent solutions of the heat equation, Manuscript, 1996.

Received March 12, 1997 and revised April 26, 1997

\section{UNIVERSITÄT ULM}

89069 ULM

Germany

E-mail address: balser@mathematik.uni-ulm.de

This work was done while being a visitor at San Diego State University, San Diego CA.. 